\title{
Diagnóstico ambiental de las áreas degradadas por la actividad minera en el municipio de Atrato, Chocó
}

\section{Environmental diagnosis of degraded areas for mining explotation in the Atrato municipality, Choco}

\author{
Lady Vargas Porras ${ }^{1}$, Zoraida Quesada ${ }^{2}$, Giovanny Ramirez ${ }^{3}$, Zulmary Valoyes ${ }^{4}$
}

RESUMEN

El municipio de Atrato cuenta con numerosas áreas de explotación minera activas y abandonadas, siendo esta actividad practicada habitualmente por foráneos en condiciones de ilegalidad. Esta situación ha generado que en la actualidad muchas de las minas activas no reciban seguimiento por parte de las autoridades competentes, lo que impide un manejo ambiental adecuado dentro del proceso productivo, causando el deterioro total de paisajes, ecosistemas y fuentes hídricas. Este estudio pretendió realizar un diagnóstico ambiental en las áreas degradadas producto de esta actividad, para lo cual se priorizó un área mediante ponderación de criterios como ubicación, extensión, cobertura vegetal y disponibilidad de agua. En esta área se realizaron análisis fisicoquímicos en muestras de agua y suelo, así como muestreos de vegetación y fauna. El diagnóstico ambiental generalizado permitió concluir que la minería genera grandes áreas deforestadas que traen consigo la emigración de especies de fauna y extinción de especies florísticas, así como la sedimentación y desviación de los cuerpos de agua utilizados en la actividad. El estudio permitió además consolidar información que sirve como base para el diseño posterior de soluciones que se ajusten a las condiciones medioambientales actuales de las áreas degradadas en éste y otros puntos de la región.

Palabras clave: Minería; Diagnóstico ambiental; Muestreo; Especies; Fuentes hidricas.

\section{ABSTRACT}

The municipality of Atrato counts on numerous active and left areas of mining operation, being this activity practiced habitually by foreign in conditions of illegality. This situation has generated that at present many of the active mines do not receive pursuit on the part of the competent authorities, which prevents a suitable environmental management within the productive process, causing the total deterioration oflandscapes, hydric ecosystems and sources. This study tried to realise an environmental diagnosis in the degraded areas product of this activity, for which an area by means of consideration of criteria was prioritized location, extension, vegetal cover and water availability. In this area physico-chemical analyses in samples of water and ground, as well as samplings of vegetation and fauna were realised. The generalized environmental diagnosis allowed to conclude that the mining generates great deforested areas that bring with himself the emigration of species of fauna and extinction of floral species, as well as the sedimentation and deviation of the used water bodies in the activity. The study allowed in addition to consolidate information
1. Ingeniera Ambiental, Especialista en Manejo Integrado de Recursos Hídricos, Contratista Instituto de Investigaciones Ambientales del Pacífico, Quibdó, Colombia. e-mail: lady8181@hotmail.com

2. Ingeniera Ambiental, Especialista en Ordenamiento y Gestión Integral de Cuencas Hidrográficas, residente ambiental, Metrocorredores Chocó, Quibdó, Colombia. e-mail:zoraidaquesada@hotmail.com

3. Biólogo, Magíster en Ciencias Biológicas (C); Investigador principal Componente Ecosistémico Instituto de Investigaciones Ambientales del Pacífico; Quibdó, Colombia.e-mail: gramirezm3@hotmail.com

4. Bióloga, Especialista en Administración de Recursos Naturales, Docente Universidad Tecnológica del Chocó; Quibdó, Colombia. e-mail: zulmaryvaloyes@hotmail.es Grupo de Investigación en Conocimiento, manejo y conservación de los ecosistemas delChocó Biogeográfico.

Recibido: 8 de marzo de 2010 Aceptado: 25 de marzo de 2010 


\section{Bioetnia Volumen 7 No 1 (enero-junio), 2010}

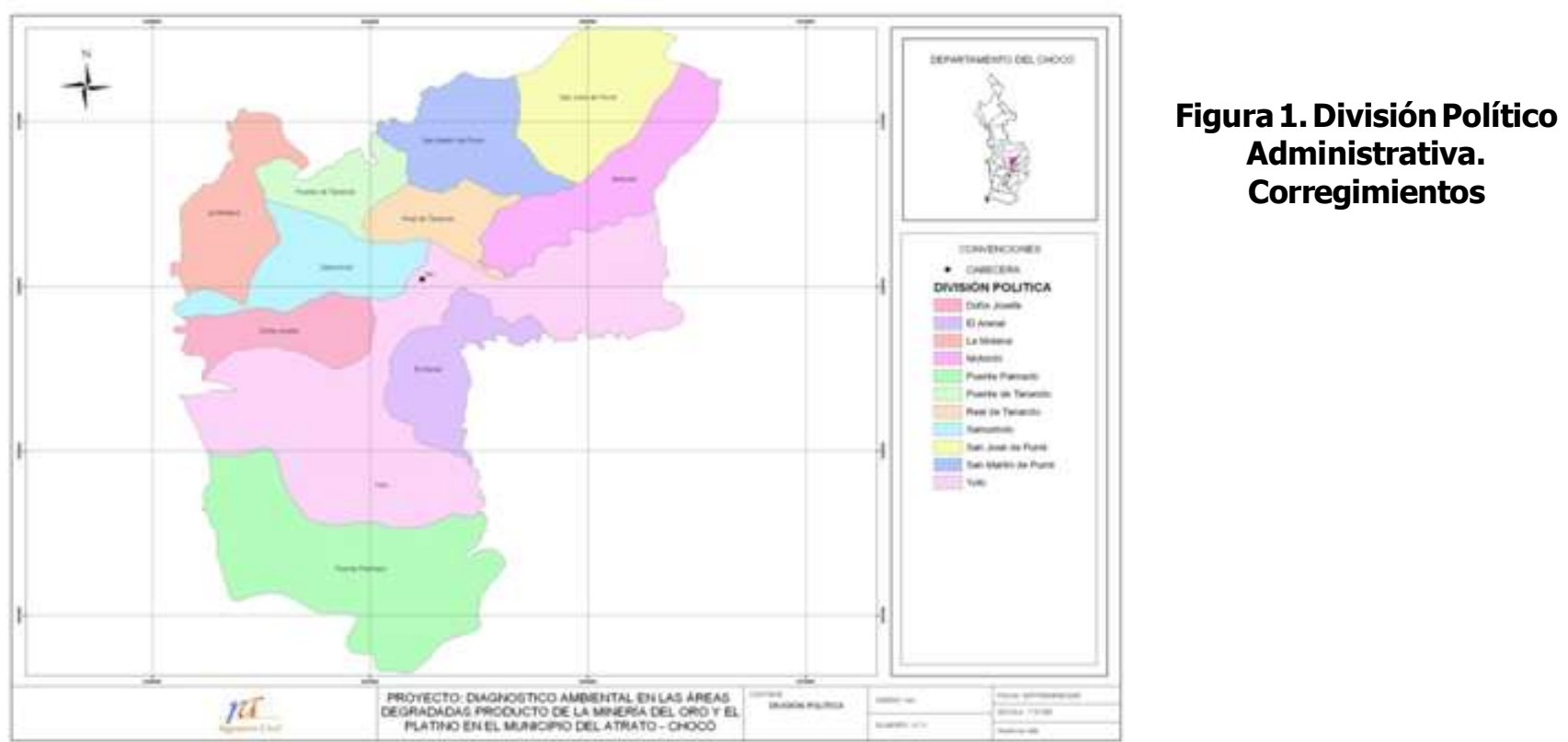

that serves as it bases for the later design of solutions that adjust to the present environmental conditions of the areas degraded in this and other points of the region.

Keyword: Mining; Environmental diagnosis; Sampling; Species; Hydric sources.

\section{INTRODUCCIÓN}

La minería a cielo abierto es una actividad industrial de alto impacto ambiental, social y cultural. Es también una actividad industrial insostenible por definición, en la medida en que la explotación del recurso supone su agotamiento. Las innovaciones técnicas que ha experimentado la minería a partir de la segunda mitad del siglo XX han modificado radicalmente la actividad, de modo que se ha pasado del aprovechamiento de vetas subterráneas de gran calidad a la explotación en minas a cielo abierto, donde se remueve la capa superficial o sobrecarga de la tierra para hacer accesibles los extensos yacimientos de mineral. Los equipos de excavación, la gran maquinaria, el uso de nuevos insumos y las tuberías de distribución permiten hoy remover montañas enteras en cuestión de horas, haciendo rentable la extracción de menos de un gramo de oro por tonelada de material removido.

La actividad minera en Colombia representa un sector importante dentro de la economía. Sin embargo, según Ramírez (2007) los procesos asociados con la minería tienen un impacto negativo en la tierra, comenzando por la exploración, etapa en la que se abren zanjas, se preparan y abren caminos y carreteables de gran envergadura para el transporte de maquinaria pesada para esta industria; hay permanentes explosiones sobre las áreas a estudiar, se destruye parte del bosque con la fauna y la flora que la contienen, se desvían ríos, quebradas, se impactan los nacederos de agua, se comienza a desplazar al poblador natural de la región, se presiona la venta de las tierras que se utilizarán en la mina y en las zonas de amortiguación.

En el Chocó, la mayor parte de la minas funciona ilegalmente, situación que dificulta el control ambiental y económico de la actividad, de ahí que la rentabilidad sea para los propietarios de la maquinaria que por lo general no son de la región. Estos factores han ocasionado que en muchos municipios como el Atrato, actualmente se puedan identificar numerosas áreas degradadas por la actividad minera que han dejado a la región ecosistemas destruidos, fuentes hídricas sedimentadas y pobreza. Con este estudio se pretendió realizar una aproximación al total de área degradada por minería en el municipio de Atrato-Chocó y un diagnóstico ambiental de las mismas, que sirva como base para que las autoridades territoriales y ambientales puedan tomar medidas tanto de control para las empresas explotadoras como de recuperación a corto y largo plazo de estas áreas.

\section{ÁREADEESTUDIO}

El municipio del Atrato está ubicado en la parte central del departamento del Chocó. Posee un área total de $725 \mathrm{~km} 2$, a una altura de $32 \mathrm{msnm}$. Dista $25 \mathrm{~km}$ de Quibdó, capital del Departamento. Con una precipitación promedio anual de $8000 \mathrm{~mm}$, una temperatura promedio de $28^{\circ} \mathrm{C}$ y situado en las coordenadas $5^{\circ} 32^{\prime}$ de latitud norte y $76^{\circ} 26^{\prime}$ de longitud oeste. Está constituido por once corregimientos y siete veredas (Alcaldía Municipal del Atrato, 2001) (Figura 1)

El municipio del Atrato en su totalidad se ubica en la 
cuenca del río Atrato, donde se han desarrollado múltiples eventos geológicos como: sedimentaciones marinas, lagunares, fluviales, intrusiones y extrusiones de rocas ígneas, represiones, transgresiones marinas y erosiones, cuyos resultados finales son los modelados geomorfológicos que se presentan en la actualidad en el territorio. Los suelos, en general, son muy superficiales a superficiales limitados por la presencia de gravilla y un nivel freático alto; las texturas son franco arenosas, francas, franco arcillosas, franco limosas y franco arcillo arenosas; la fertilidad por lo general es baja, en algunos casos puede ser moderada y en otros casos, alta. Estos suelos son los que más se utilizan con cultivos de subsistencia, tales como: arroz, plátano, yuca, caña, achiote, borojó, almirajó, bacao, pacó. El arroz, en algunas zonas, da buenos rendimientos con aplicación de fertilizantes completos. Se deben hacer zanjas de drenaje y conservar la vegetación ribereña como protección ante el efecto erosivo ocasionado por los ríos. El municipio del Atrato, desde el punto de vista hídrico cuenta con abundantes cursos de agua representados en ríos y quebradas, entre los que se encuentran $\mathrm{s}$ el río Atrato, sus afluentes y otras cuencas hidrográficas como el río Tanandó, río Samurindó, río Purré, río Yuto, río Paimadó, quebrada El Rosario, quebrada Tocolloró y la quebrada Doña Josefa (Alcaldía Municipal de Atrato, 2001).

En cuanto al componente vegetal las principales especies arbóreas que se encuentran en el municipio son: el lechero (Ambelania sp), cedro (Cederia angustifonoi), guácimo (Luchea simanil), aceite (Calophyllum mariae), jigua (Beilschmiedia rohliana), carrá, (Huberodendro patinoi), caimito (Pouteria sp), abarco (Cariniana periformes), chanó (Sacoglotis procerum), guamo (Inga sp), roble (Tabebuia rosea), algarrobo (Hymenea palustris), sandé (Brasium utile), peine de mono (Apeiba membranaceae), ají (Duguetia sp), lirio (Couma macrocarpa), aliso (Simarouba amara), algodoncillo (Hampea sp), palma don pedrito (Oenacarpus mapora), guarumo (Cecropia sp), guayabillo (Caycophyllum candidissimun), palo perico (Persea sp), insibe (Amiba sp), níspero (Chrysophyllum sp), jabóncillo (Isetia pittieri), caracolí o espave (Clorophora tinctoria) y el costillo (Sagotia racemosa), entre muchas otras. La zona también presenta gran variedad de fauna silvestre entre las que se pueden mencionar especies como mico (Cebus vestias), guatin (Dasy procta puntata), tatabro (Tayassu tajacú), guagua (Agouti paca), amarillo (Dasypusnovemcinctus), guagua lapa (Agouti paca), iguana verde (Iguana iguana) y perdiz (Cjoloepustetradoctula), entre otras registradas para elárea (CORPOICADNP-FONADE, 1998).

El municipio del Atrato, está constituido en su mayor parte por llanuras y terrenos selváticos, con relieves ondulados y algunos sectores quebrados que denotan un proceso de disección fuerte y generalizado (Arango y Saldarriaga, 1997). Desde el punto de vista hidrográfico cuenta con abundantes cursos de agua en forma de ríos y quebradas, que se convierten en una de las principales vías de comunicación de sus habitantes. La vegetación que predomina en el municipio es el bosque húmedo tropical que se encuentra en la mayor parte del territorio que baña el río Atrato y en las partes altas hay gran variedad de bosques montañosos. La precipitación promedia anual en las estaciones utilizadas es de $8000 \mathrm{~mm}$ aproximadamente. Los meses más secos son: enero, febrero, marzo y diciembre y los meses más húmedos comprenden los meses entre junio y septiembre. Se registran temperaturas medias anuales que oscilan entre $25.9^{\circ} \mathrm{Cy} 26.7^{\circ} \mathrm{C}$, temperaturas máximas promedias anuales que oscilan entre $33.8^{\circ} \mathrm{C}$ y $34.9^{\circ} \mathrm{C}$ y temperaturas mínimas promedias anuales que oscilan entre $20.7^{\circ} \mathrm{Cy} 22^{\circ} \mathrm{C}$.

Las características físicas y químicas de los suelos garantizan el desarrollo sostenible de las especies vegetales que sobre él habitan. Sin embargo, muchas de éstas han sido afectadas y modificadas debido al desarrollo de actividades mineras tecnificadas que ocasionan pérdida y degradación del suelo en grandes áreas del municipio.

Las principales actividades económicas del municipio del Atrato corresponden al sector primario de la economía; sobresalen la agricultura, la minería y la extracción de madera. La base de producción agrícola del municipio está dada por los cultivos de plátano, maíz, yuca, ñame, banano y frutales (piña, borojó, aguacate, papaya, lulo, guayaba, caimito, arroz, etc.). Esta producción en su mayoría es para consumo interno y algunos excedentes se comercializan en Quibdó. La actividad pecuaria es en especial de aves, cría de cerdo y de manera muy escasa la explotación bovina, estas actividades se realizan con técnicas tradicionales, se destaca que es una actividad de autoconsumo con muy baja comercialización. La explotación forestal y la pesca son actividades sobresalientes. La explotación de oro y platino en el municipio del Atrato, se realiza tradicionalmente con métodos artesanales y en ocasiones mediante el empleo de motobombas, pero en los últimos años, foráneos han introducido la práctica de esta actividad de manera tecnificada, ocasionando un deterioro al medio ambiente, los recursos naturales y pocas oportunidades laborales para los mineros artesanales (CORPOICA-DNP-FONADE, 1998).

\section{METODOLOGÍA}

Ubicación, extensión, estado de la cobertura vegetal, disponibilidad de agua y tiempo de intervención. Para el establecimiento de la ubicación geográfica precisa de las áreas degradadas por la minería en el municipio del Atrato, se llevó a cabo un recorrido por las áreas mineras abandonadas, localizadas en los corregimientos de Yuto, Doña Josefa, Tanandó, Samurindó, Paimadó, La Molana y Vuelta Manza. Este recorrido permitió la generación de cartografía con la 


\section{Bioetnia Volumen 7 № 1 (enero-junio), 2010}

localización de las mismas, así como la determinación del estado actual de estas áreas.

La extensión de las diferentes áreas se determinó mediante el uso de un GPS programado para la toma de coordenadas cada 10 segundos, haciendo un recorrido por el borde de cada zona, teniendo en cuenta las limitaciones morfométricas existentes. Esta información se procesó mediante Sistemas de Información Geográfica-SIG para obtener el cálculo de áreas. El establecimiento del estado de la cobertura vegetal, la disponibilidad de agua y el tiempo de intervención, se llevó a cabo mediante observación directa y con la ayuda de entrevistas a miembros de la UMATA y personas de la comunidad.

Priorización. En la priorización se utilizó información generada a partir de observaciones in situ del equipo técnico y el desarrollo de entrevistas a personas locales; con base en esta información se ponderaron las áreas, teniendo en cuenta los siguientes criterios: ubicación, extensión, estado de la cobertura vegetal, disponibilidad de agua y tiempo de intervención. En el marco de esta ponderación de criterios, se dio un valor a cada uno por área evaluada en la escala de 1 a 5 ; al final se organizaron estas áreas en orden de prioridad, de acuerdo con el puntaje total obtenido.

La ubicación se refiere a la distancia existente entre la zona degradada y el casco urbano del municipio en estudio, y está directamente relacionada con las condiciones de ingreso a la zona y la facilidad de transporte hacia ella. Para establecer la calificación correspondiente a cada área en este aspecto se utilizaron los siguientes rangos: De 0 a $2 \mathrm{~km}$ con una calificación de 5 puntos, de $2 \mathrm{~km}$ a $5 \mathrm{~km}$ se obtiene un calificación de 4 puntos, para una distancia de $5 \mathrm{~km}$ a $8 \mathrm{~km}$ se obtiene una calificación de 3 puntos, de 8 km a $11 \mathrm{~km}$ la calificación será de 2 puntos y para más de $11 \mathrm{~km}$ de distancia solo se obtendrá un punto.

La extensión se refiere al área cubierta por la zona degradada por la minería; este valor se determinó mediante el uso de SIG. Para establecer la calificación correspondiente a cada área, en este aspecto se utilizaron los siguientes rangos: un punto de calificación para un área de $0 \mathrm{a} 2$ ha, dos puntos para un rango de 2 ha a 6 ha, tres puntos para áreas que van desde 6 ha a 8 ha, cuatro puntos si el tamaño oscila entre 8 ha y 10 ha y 5 puntos de calificación para más de 10 ha.

El estado de la cobertura vegetal se refiere al estado actual de crecimiento de vegetación o auto-regeneración de la zona. La calificación para cada área se obtuvo mediante observación directa, dando a cada situación una calificación de la siguiente manera: 5 puntos para zonas donde no se encontró vegetación, 4 puntos para áreas en las cuales se estaba iniciando crecimiento de rastrojos, 3 puntos para zonas con crecimiento medio, 2 puntos para aquellas áreas donde existen procesos de reforestación en desarrollo y un punto para áreas totalmente reforestadas.
El criterio de disponibilidad de agua se refiere la cercanía a fuentes hídricas naturales o modificadas, que puedan servir como abastecedoras de agua para riego en caso de que se establezcan medidas de recuperación con siembras de alguna especie. La calificación se hizo de acuerdo con los rangos siguientes: 5 puntos para aquellas áreas que presentan fuentes hídricas a menos de $2 \mathrm{~m}, 4$ puntos si la fuente hídrica se encuentra entre $2 \mathrm{~m}$ y $7 \mathrm{~m}, 3$ puntos en caso de que la fuente se encuentre entre $7 \mathrm{~m}$ y $12 \mathrm{~m}, 2$ puntos para los casos en que la fuentes disponible de agua se encuentre entre $12 \mathrm{n}$ y $17 \mathrm{~m}$ y solo un punto si esta se encuentra a más de $17 \mathrm{~m}$.

El criterio de tiempo de intervención se refiere al lapso de tiempo que lleva el área desde su última explotación minera. La calificación se hizo de acuerdo con los siguientes rangos: Para las áreas con explotación reciente de 0 a 3 meses se calificó con 5 puntos, de 3 meses a 6 meses con 4 puntos, de 6 meses a 9 meses con 3 puntos, de 9 meses a 1 año con 2 puntos y más de un año con 1 punto.

Para la priorización se establecieron los siguientes porcentajes de importancia para cada criterio: la ubicación del área equivale a un $20 \%$ de importancia, la extensión al 10\%, la cobertura vegetal representa un $25 \%$, la disponibilidad de agua equivale al $20 \%$ y el tiempo de la última intervención equivale a $25 \%$. Así se tiene para cada área una calificación total calculada de la siguiente manera:

$$
\begin{gathered}
\text { Calificación Total }=\text { CT } \\
\text { CT }=(\text { valor de calificación de ubicación*0.20) }+ \\
(\text { valor de calificación de extensión*0.10) }+ \\
\left(\text { valor de calificación de cobertura vegetal }{ }^{*} 0.25\right)+ \\
(\text { valor de xalificación de disponibilidad de agua* } 0.20)+
\end{gathered}
$$

(valor de calificación de tiempo de intervención*0.25)

Como resultado de estos cálculos se obtiene el área priorizada de acuerdo con el máximo valor de calificación total obtenida, en la cual se tomarán las muestras de suelo, agua y vegetación, para el diagnóstico ambiental. Finalmente se determinaron rangos de prioridad de recuperación de área dependiendo del valor obtenido, es decir que se identificaron las áreas con mayor urgencia de implementación de alternativas de recuperación, estableciendo un rango de prioridad alto de 4-5, medio de 2.8-3.9 y bajo de 0-2.7.

Diagnóstico ambiental. El diagnóstico ambiental se realizó teniendo en cuenta los análisis fisicoquímicos realizados a muestras de agua y suelo, y los resultados del muestreo aleatorio de vegetación realizado en el área priorizada (Figura 2).

Para la caracterización del componente hídrico se tomaron nueve muestras de agua, en diferentes puntos (Tabla 1). En cada una se analizaron los parámetros de sólidos totales, sólidos suspendidos, sólidos sedimentables, sólidos disueltos, oxígeno disuelto, fosfatos, nitritos, nitratos, sulfatos, acidez y alcalinidad, análisis que se realizarán en el laboratorio 


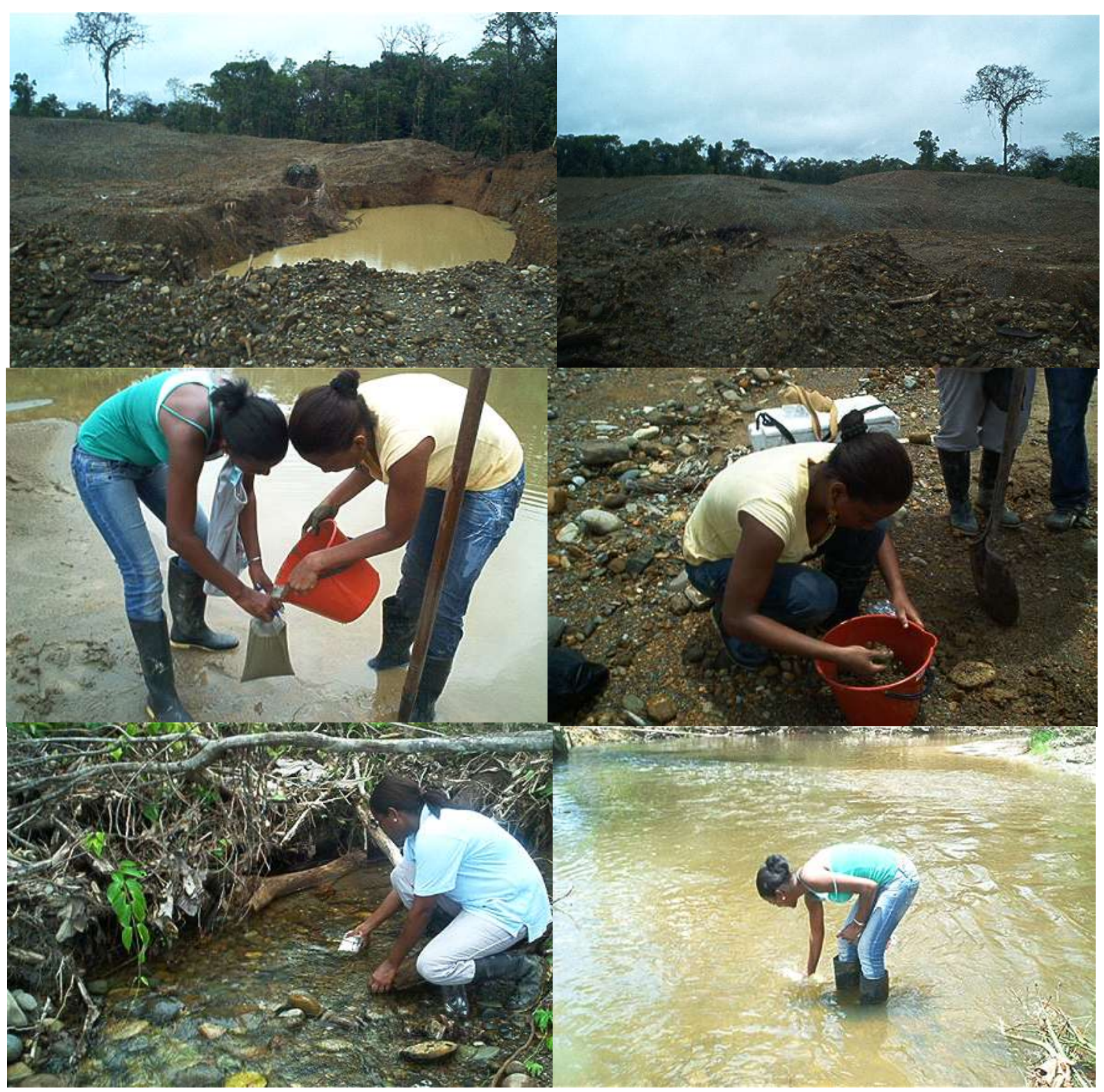

Figura 2. Área priorizada y muestreos de suelo y agua.

certificado de CODECHOCO.

Se tomaron siete muestras de suelo incluyendo dos de lodos provenientes de los pozos generados en la actividad minera, a las que se les analizaron los parámetros de mercurio, $\mathrm{pH}$, potasio, fosforo, calcio, intercambio de iones catiónicos y porcentaje de humedad, en el laboratorio de suelos de la Universidad Nacional de Medellín y se realizó un muestreo aleatorio de vegetación circundante para identificar especies predominantes en la zona y establecer la composición del bosque antes de la explotación minera. La Figura 3 muestra el área priorizada, El colegio y la distribución de puntos de muestreo de agua y suelo.

El diagnóstico ambiental se desarrolló a partir del análisis comparativo entre las características iniciales de este tipo de ecosistemas y el estado actual de sus recursos. Para realizar una aproximación exacta se tuvieron en cuenta los resultados de las pruebas físicoquímicas en el recurso agua y suelo, así como la estructura y composición del esqueleto vegetal de las 
Bioetnia Volumen 7 № 1 (enero-junio), 2010

Tabla 1

Puntos de muestreo

\begin{tabular}{|c|c|c|c|}
\hline \multirow[t]{2}{*}{ Punto } & \multirow[t]{2}{*}{ Lugar } & \multicolumn{2}{|c|}{ Coordenadas } \\
\hline & & $\mathbf{x}$ & $\mathbf{Y}$ \\
\hline \multirow[t]{2}{*}{ Muestra de agua 1} & QUEBRADA DOÑA JOSEFA AGUAS & & \\
\hline & ABAJO DEL ÁREA DE LA MINA EL COLEGIO & 1048259 & 1103731 \\
\hline Muestra de agua 2 & POZO DE AGUA 3 & 1048267 & 1103801 \\
\hline Muestra de agua 3 & POZO DE AGUA 4 & 1048140 & 1103917 \\
\hline Muestra de agua 4 & BRAZO UNO DE LA QUEBRADA AGUA CLARA & 1048187 & 1103460 \\
\hline Muestra de agua 5 & BRAZO DOS DE LA QUEBRADA AGUA CLARA & 1048225 & 1103450 \\
\hline \multirow[t]{2}{*}{ Muestra de agua 6} & INTERSECCION QUEBRADA DOÑA JOSEFA CON & & \\
\hline & BRAZO UNO DE QUEBRADA AGUA CLARA & 1048211 & 11603586 \\
\hline \multirow[t]{2}{*}{ Muestra de agua 7} & INTERSECCIÓN QUEBRADA DOÑA JOSEFA CON & & \\
\hline & BRAZO DOS DE QUEBRADA AGUA CLARA & 1048217 & 1103535 \\
\hline \multirow[t]{2}{*}{ Muestra de agua 8} & QUEBRADA DOÑA JOSEFA AGUAS ARRIBA DEL & & \\
\hline & ÁREA DE LA MINA EL COLEGIO & 1048322 & 1103439 \\
\hline Muestra de agua 9 & AGUA NATURAL SIN CONTAMINANTES DE MINERIA & \multicolumn{2}{|c|}{ Quebrada Villa Luz } \\
\hline Muestra de suelo 1 & LODO DE POZO DE AGUA 3 & 1048267 & 1103801 \\
\hline \multirow[t]{2}{*}{ Muestra de suelo 2} & SUSTRATO RESULTANTE DEL LAVADO DE TIERRA & & \\
\hline & DE LA ACTIVIDAD MINERA & 1048213 & 1103776 \\
\hline Muestra de suelo 3 & LODO DE POZO DE AGUA 4 & 1048140 & 1103917 \\
\hline \multirow[t]{2}{*}{ Muestra de suelo 4} & SUSTRATO RESULTANTE DEL LAVADO DE TIERRA & & \\
\hline & DE LA ACTIVIDAD MINERA & 1048187 & 1103460 \\
\hline \multirow[t]{2}{*}{ Muestra de suelo 5} & SUSTRATO RESULTANTE DEL LAVADO DE TIERRA & & \\
\hline & DE LA ACTIVIDAD MINERA & 1048200 & 1103449 \\
\hline \multirow[t]{2}{*}{ Muestra de suelo 6} & SUSTRATO RESULTANTE DEL LAVADO DE TIERRA & & \\
\hline & DE LA ACTIVIDAD MINERA & 1048210 & 1103516 \\
\hline Muestra de suelo 7 & SUELO DE BOSQUE LIBRE DE MINERÍA & 1048509 & 1103383 \\
\hline
\end{tabular}

áreas circundantes; para la aproximación al estado actual de la fauna se tuvieron en cuenta, testimonios de cazadores que frecuentaron el área de estudio. Lo anterior permitió determinar los impactos generados al ecosistema por la actividad minera en el municipio de Atrato.

\section{RESULTADOSY DISCUSIÓN}

Se georreferenciaron 30 áreas con procesos de degradación, que presentaron condiciones muy similares en algunos criterios; cabe anotar que estas zonas no representan el total de las existentes en el municipio porque por motivos de seguridad no se visitaron algunos corregimientos como San Martín, San José, Motoldó y Real Tanandó (Tabla 2 y Figura 3).

Como resultado de las observaciones y la información primaria levantada por el equipo técnico se determinaron las calificaciones por criterio para cada área visitada y se obtuvo la zona denominada ElColegio como el área priorizada, en ella se realizaron todos los análisis fisicoquímicos de agua, pruebas de fertilidad y mercurio en suelo y muestreos aleatorios de vegetación circundante para determinar la composición del bosque antes de la explotación minera, información que permitió la realización del diagnóstico ambiental del área.

Componente agua. En términos generales, este componente es uno de los más afectados por la actividad minera, porque en casi todas las fases del proceso minero se utilizan los ríos, riachuelos y quebradas como fuentes únicas de abastecimiento y disposición; de allí que esta actividad sea la causante de los impactos más severos sobre los cuerpos de agua del municipio del Atrato, que están relacionados con la modificación de la estructura de las redes hídricas locales. En este sentido se lograron evidenciar fuertes desviaciones de cauces, gran cantidad de represamientos de cuerpos de aguas 
Tabla 2 Áreas degradadas por corregimiento

\begin{tabular}{|c|c|c|}
\hline Número & Área & Corregimiento \\
\hline 1 & CATANGUERA & YUTO \\
\hline 2 & LA EMILIA & \\
\hline 3 & YUTO 1 & \\
\hline 4 & DOÑA ROSA 1 & \\
\hline 5 & DOÑA ROSA 2 & \\
\hline 6 & DOÑA ROSA 3 & \\
\hline 7 & EL PEÑÓN 1 & \\
\hline 8 & EL PEÑÓN 2 & \\
\hline 9 & YUTO NUEVO & \\
\hline 10 & YUTO VIEJO & \\
\hline 11 & YUTO VIEJO2 & \\
\hline 12 & YUTO VIEJO 3 & \\
\hline 13 & RANCHERÍA PARTE ALTA & \\
\hline 14 & LA EMILIA 2 & \\
\hline 15 & LA TECLA 1 & \\
\hline 16 & LA TECLA2 & \\
\hline 17 & LA POZA & \\
\hline 18 & FIGUDA & \\
\hline 19 & COLEGIO & \\
\hline 20 & MANDINGA TANANDÓ & TANANDÓ \\
\hline 21 & LA PORTUGUESA & \\
\hline 22 & DOÑA JOSEFA & DOÑA JOSEFA \\
\hline 23 & GUARIPITA & ARENAL \\
\hline 24 & GUARAPITA 2 & \\
\hline 25 & PUENTE & SAMURINDÓ \\
\hline 26 & MINA GRANDE & \\
\hline 27 & MIRADOR DE LA ROSA & \\
\hline 28 & LA MOLANA & LA MOLANA \\
\hline 29 & VUELTA MANZA & \\
\hline 30 & PUENTE PAIMADO & PAIMADO \\
\hline
\end{tabular}

lóticas, también se afectaron los procesos naturales de escorrentías debido a la modificación del relieve natural del suelo y una alta sedimentación debido al aporte de sólidos procedentes del lavado de los sustratos del suelo, además de una disminución acelerada de la oferta hídrica de la zona. Es importante resaltar que la naturaleza de este componente no permite hacer un uso sectorizado del mismo; con esto se provoca la contaminación no sólo de la fuente directa sino también la de todos los elementos que se encuentran adjuntos a esta red; la afectación se hace a una escala de tiempo tan corta y con una intensidad tan alta que les es casi imposible a estos sistemas hídricos activar la capacidad natural de resiliencia, agravando aún más la situación.

Además de los impactos en la estructura, se generan impactos en la composición fisicoquímica del agua; lo que afecta variables como sólidos, turbiedad, alcalinidad, $\mathrm{pH}$, que determinan la calidad del recurso para la preservación de la vida acuática. Esto se pudo determinar luego del análisis de parámetros fisicoquímicos en los diferentes puntos de muestreo, que son una referencia para determinar el tipo de recuperación que estos cuerpos de agua pueden tener.

Para el análisis del impacto ocasionado por la actividad minera en el componente hídrico se analizaron muestras de agua en diferentes puntos del área El Colegio, que incluye quebradas y pozos; adicionalmente se tuvo en cuenta la quebrada Villa Luz en la que no se practica esta actividad extractiva y servirá como base para comparar los resultados entre una fuente natural y una intervenida por la minería.

La presencia de oxígeno en el agua es indispensable para la vida de peces y otros seres acuáticos; por tanto, su análisis es una prueba clave en la determinación de la contaminación del agua. Un nivel de oxígeno disuelto de $5 \mathrm{mg} / \mathrm{l}$ o superior ayuda al crecimiento favorable de la mayoría de especies de peces en un cuerpo de agua (http://www.hannaarg.com). De acuerdo con los resultados de los análisis de laboratorio obtenidos, los puntos de muestreo presentan un nivel de oxígeno aceptable, con concentraciones promedio que varían desde 4.22 hasta $7.22 \mathrm{mg} / 1$; se destacan los valores más bajos en los pozos generados durante la extracción de oro y los valores más altos en la parte aguas arriba de la quebrada Doña Josefa y en la quebrada Villa Luz, esto debido sobre todo a que en esa zona no se presentan descargas de la actividad minera, lo que también sucede con la Quebrada Villa Luz. Es importante destacar que en esta quebrada, se realizan actividades de origen doméstico por parte de los habitantes de la zona, lo que influye en la cantidad de oxígeno disuelto presente en la misma.

En términos generales las fuentes hídricas muestreadas presentaron unas concentraciones normales de nitratos, nitritos y fosfatos, sobre todo porque no existe contaminación por materia orgánica y porque en este tipo de fuentes los nutrientes se encuentran asociados con la vida acuática y atrapados en la biomasa bentónica o en el sedimento, que han sido removidos por la intervención minera. El contenido en nitratos, nitritos y fosfatos permite una valoración de la calidad del agua y proporciona información sobre problemas medioambientales como la eutrofización o carencia de oxígeno en el agua. La norma indica $25 \mathrm{mg} / \mathrm{l}$ como valor de calidad para los nitratos, y una concentración máxima de nitratos y nitritos de $45 \mathrm{mg} / 1$ y $0.1 \mathrm{mg} / \mathrm{l}$, respectivamente (Stumm y Morgan 1988). Aunque el fósforo y el nitrógeno se consideran nutrientes esenciales, los niveles encontrados en las fuentes hídricas de fosfatos son mínimos, lo que indica 


\section{Bioetnia Volumen 7 № 1 (enero-junio), 2010}

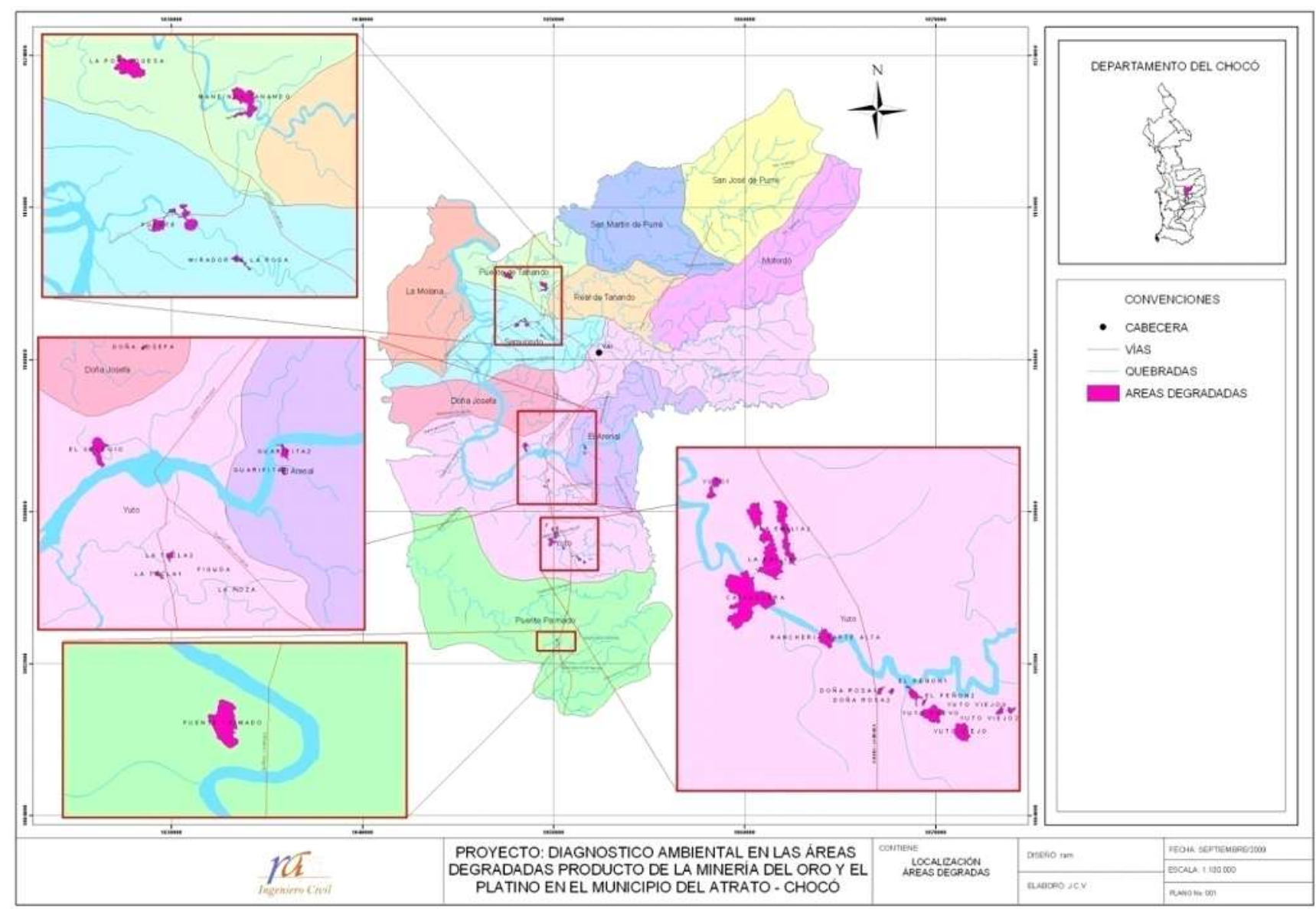

Figura 3. Distribución de áreas degradadas visitadas por corregimiento

mínimo desarrollo de vida acuática al interior de éstas.

La alcalinidad se entiende como la capacidad para neutralizar ácidos y la acidez la capacidad de neutralizar bases; la medición de estos parámetros en los puntos de muestreo permitió medir la preponderancia de cada tipo de sustancia y como se observa en los resultados, los cuerpos de agua de la mina El Colegio son sobre todo de carácter ácido, con un rango entre 13.6 y $23.3 \mathrm{mg} / \mathrm{l}$, presentando la concentración más alta en la intersección de la quebrada Doña Josefa con la quebrada Agua Clara, en la que se estaba practicando extracción de metales por barequeo durante el muestreo, lo que justifica el resultado, teniendo en cuenta que el principal origen de la acidez en las agua naturales se debe al $\mathrm{CO}_{2}$ y a sales hidrolizadas de algunos metales que son residuos de la actividad minera, de ahí que los puntos de muestreo ubicados en los pozos presentan también altas concentraciones de este parámetro, porque por su carácter lentico no presentan recirculación que minimice estos niveles.

El ion sulfato es uno de los que se presenta en mayor cantidad en aguas naturales. Todas las aguas naturales contienen sulfatos que provienen de yeso y minerales simi- lares. Su determinación es importante porque produce efectos característicos sobre las personas cuando su concentración es alta. Las concentraciones de sulfatos en el agua muestreada presentan un rango de 2.0 a $11.09 \mathrm{mg} / 1 ;$ los niveles más altos se encuentran en el pozo 4 debido a los efluentes de la actividad minera que se concentran en este tipo de fuentes.

El mayor impacto de la actividad minera en la composición fisicoquímica del agua, es la concentración de sólidos, como consecuencia de la remoción en masa, la pérdida de suelo, la afectación de la estabilidad y de la estructura del mismo que genera esta actividad, lo que finalmente desencadena un proceso de sedimentación de cuerpos de agua, debido, además, a procesos de excavación, vertimiento de aguas usadas, lavado de material, lavado de lodos y desprendimiento de peñas. Las concentraciones de sólidos totales y sólidos sedimentables son considerables en los puntos de muestreo, y elevadas en algunos puntos como en los pozos y en la intersección de las quebradas Aguas Claras y Doña Josefa, donde se presentan altos niveles de este parámetro, porque al momento del muestreo se observó a personas de la comu- 


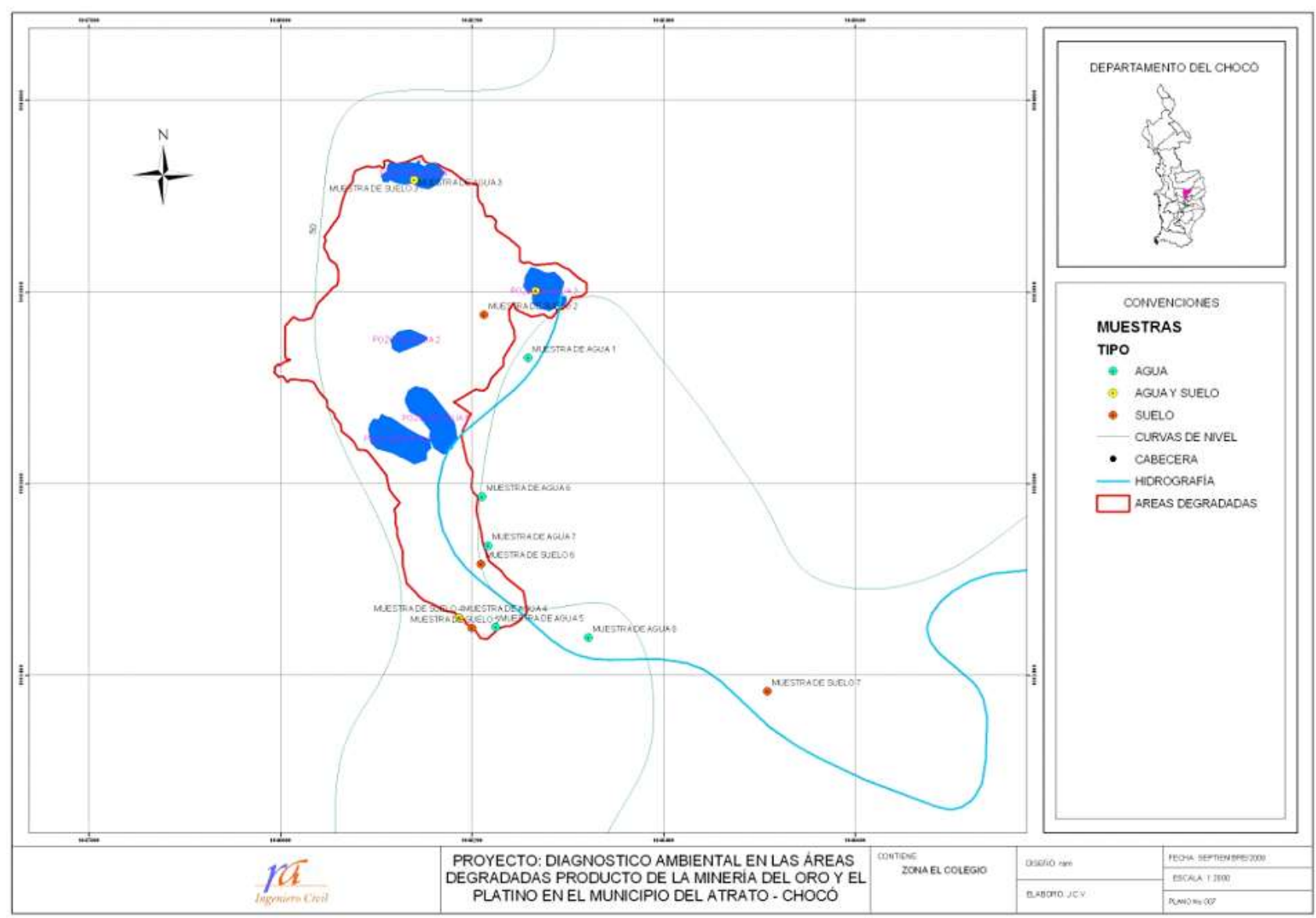

Figura 4. Área El Colegio y puntos de muestreo.

nidad realizando actividades mineras artesanales (barequeo). Es importante mencionar que la diferencia entre los sólidos sedimentables y disueltos es muy alta, lo que muestra que los sólidos presentes en las fuentes hídricas del área de estudio son sobre todo material suspendido generado por las acciones mencionadas.

Componente suelo. Los resultados del análisis foliar y las pruebas de fertilidad realizadas en los siete puntos de muestreo escogidos, que incluyeron textura $\mathrm{pH}$, materia orgánica, fósforo, calcio, magnesio, potasio, aluminio, capacidad de intercambio catiónico efectivo, manganeso, cobre, zinc, azufre, hierro y boro. Estos mostraron la predominancia de suelos franco arcilloso, arenoso y limoso, porque presentan en su mayoría un porcentaje superior de arena en el caso de sustratos de lodos de sedimentación de pozos dejados por la actividad minera y de limos en los sectores más altos del área. Esta situación se presenta notablemente en los seis primeros puntos de muestreo ubicados dentro del área degradada, que a diferencia del punto siete corresponde a suelo de bosque remanente que muestra una mezcla de arenas, arcillas y materia orgánica (Figura 4).
En este contexto se observa que el impacto generado por la extracción de metales en el suelo, es sobre todo la pérdida de fertilidad, que está directamente relacionada con la presencia de diferentes sustratos y materia orgánica que permita el desarrollo de especies, esto debido al lavado que se realiza en los horizontes de este suelo, donde es el agua la encargada de arrastrar y retirar los nutrientes, que se sedimentan en pozos o van directamente a las fuentes hídricas de la zona, de ahí que los sustratos encontrados en el área sean casi en su totalidad arenas en las partes altas y arcillas en las zonas bajas. Esto implica no sólo un impacto negativo al suelo, sino también a las especies vegetales que dependen de él para su crecimiento y a las fuentes hídricas que además de ser desviadas sufren procesos de sedimentación al recibir grandes descargas de sólidos, que interfieren con el desarrollo de la vida acuática y el uso doméstico.

El pH además de expresar la presencia de iones hidrógeno que determinan el nivel de acidez o basicidad del suelo, influye en el grado de solubilidad de minerales y nutrientes por parte del mismo, de ahí la importancia de medición de este parámetro porque la vegetación sólo puede absorber a los minerales 
Bioetnia Volumen 7 № 1 (enero-junio), 2010

Tabla 3

Especies observadas en el bosque aledaño al área degradada por minería "El Colegio"

\begin{tabular}{|c|c|c|c|}
\hline $\mathbf{N}^{\circ}$ & Familia & Nombre científico & Nombre vulgar \\
\hline 1 & Annonaceae & Xilopia cf polyantha & \\
\hline 2 & Araliaceae & Sheflera sp & \\
\hline 3 & Araceae & Anthurium cf chlorocarpum sódica & \\
\hline 4 & & Anthurium cf vallensis & \\
\hline 5 & & Anthurium garagaranum stand & \\
\hline 6 & & Anthurium paludasum & \\
\hline 7 & & Anthurium sodirognum engle & \\
\hline 8 & & Philodendrum aff omatum scnott & \\
\hline 9 & & philodendrum roseo & \\
\hline 10 & & Philodendronlongipedunculatum & \\
\hline 11 & Arecaceae & Bactris sp & \\
\hline 12 & & Euterpe oleoracea & Naidi \\
\hline 13 & & Geonoma $\mathrm{sp}^{1}$ & \\
\hline 14 & & Geonoma $\mathrm{sp}^{2}$ & \\
\hline 15 & & Maurutiella macroclada & Quita sol \\
\hline 16 & & Oenacarpus mapora & Don Pedrito \\
\hline 17 & & Oenocarpus batawa & Mil pesos \\
\hline 18 & & Socratea exorrhiza & Sancona \\
\hline 19 & & Wetinia quinaria & Meme \\
\hline 20 & & Wetinia $s p$ & \\
\hline 21 & Bombacaceae & Huberodendrun patinoi & Carra \\
\hline 22 & & Matisia castano & Sapote monte \\
\hline 23 & & Matisia lectandra & \\
\hline 24 & Bromeliaceae & Gusmania sp & \\
\hline 25 & & Gsmania cf linguilata & \\
\hline 26 & Burseraceae & Protium $\mathrm{sp}$ & \\
\hline 27 & Caesalpinaceae & Protium venerolense & \\
\hline 28 & & Hymenea palustris & Algarrobo \\
\hline 33 & & Clusiasp & Matapalo \\
\hline 34 & & Vismia ferruginea & Sangre de toro \\
\hline 35 & & Vismia macrophylla & Manchara \\
\hline 36 & Eufhorbiaceae & Croton chocoensis & \\
\hline 37 & Fabaceae & Inga sp & Guamo \\
\hline 38 & Gesneriacea & Columnea consanguinea & \\
\hline 39 & & Paradrimoniasp & \\
\hline 40 & Lecithydaceae & Gustavia superba & \\
\hline 41 & Maranthaceae & Calathea sp & \\
\hline 42 & Melastomataceae & Miconia sp1 & \\
\hline 43 & & Miconia sp2 & \\
\hline
\end{tabular}


Áreas degradadas por la minería en el Atrato. L Vargas et al.

Tabla 3

Especies observadas en el bosque aledaño al área degradada por minería "El Colegio" (continuación)

\begin{tabular}{llll}
\hline No & Familia & Nombre científico & Nombre vulgar \\
\hline 44 & & Osaea bracteata & \\
45 & & Topobea parasítica & \\
46 & & Tococa guianensis & \\
47 & Meliaceae & Cedrela odorata & Cedro \\
48 & Mimosaceae & Albizziasp & \\
49 & & Caliandrasp & Acacia \\
50 & & Calliandraglaberrima & \\
51 & Monimiaceae & Siparuna guianensis & \\
52 & Moraceae & Brosimum sp & \\
53 & & Brosimum utile & \\
54 & & Fincus $s p$ & Casaco \\
55 & Ochnaceae & Cespedesia spathulata & Caimo \\
56 & Sapotaceae & Pouteria sp & Peine mono \\
57 & Tiliaseae & Apeiba aspera &
\end{tabular}

disueltos. El pH de la mayor parte de los suelos varía entre 4 y 8 , pero el rango óptimo para el crecimiento de la mayor parte de los vegetales es de 6 a 7 porque la mayoría de las sustancias nutritivas de las plantas están disponibles en este intervalo; de acuerdo con ello el área muestreada presenta buen comportamiento en cuanto a este parámetro pues los valores obtenidos oscilan entre 4.6 y 6.3 .

La presencia de materia orgánica y de sustancias como calcio, aluminio y magnesio es mínima, presentándose las mayores concentraciones en el punto cuatro de muestreo, donde se observó un sustrato mezclado y se evidenció la presencia de restos de madera, remanente de raíces de árboles y mayor humedad por la cercanía a la quebrada Agua Clara. En cuanto al intercambio catiónico que expresa la capacidad de absorber y cambiar cationes de nutrientes, y que aumenta con el contenido de materia orgánica, se observa que los valores obtenidos para todos los puntos de muestreo oscilan entre 1 y 8.4 , encontrándose el valor más alto igualmente en el punto 4 por la situación descrita anteriormente, lo que explica también los valores bajos obtenidos en las demás zonas carentes de materia orgánica.

El análisis foliar de las muestras incluye la determinación de las concentraciones de fósforo, azufre, magnesio, hierro, cobre, zinc y boro. Aunque el hierro es uno de los elementos más frecuentes en las rocas y en los suelos se observa una gran diferencia en la concentración de este elemento si se compara el valor obtenido para suelo de bosque sin actividad minera en el punto siete de muestreo, con los valores obtenidos en las demás muestras, lo que evidencia nuevamente el impacto de esta actividad en la presencia de nutrientes, porque estos son retirados durante el proceso de extracción de metales alterando la carga eléctrica y modificando las propiedades de superficie del mismo y su capacidad amortiguadora. En la parte alta se encuentra el manganeso que por proceso de meteorización y escorrentía se arrastra hacia la parte baja, inundando los suelos de los valles aluviales de este elemento, lo que explica la presencia en grandes cantidades en el punto de muestreo uno.

También se analizó la concentración de mercurio en las muestras tanto de suelo como de lodo proveniente de los pozos, este contaminante es altamente peligroso por sus efectos en la salud humana y por su condición bioacumulable que lo convierte en un elemento persistente en la cadena trófica una vez ingresa a ella. El análisis de este parámetro es un factor determinante en el diseño de alternativas de recuperación; los resultados indican que las muestras de suelo y lodo tomadas en el área El Colegio no presentan concentraciones de mercurio, lo que sugiere una movilización del mismo a otras zonas y el requerimiento de más muestreos para su determinación.

La suma de las variables como estrucutura y composición del suelo, muestran que para una recuperación del mismo, se hacen necesarios procesos como adecuación de terrenos porque por la práctica de la actividad minera se encuentran distribuidos de forma irregular. Es necesario además, construir una red de drenaje que permita la evacuación de grandes cantidades de agua retenidas en los mismos. Asimismo se requiere la recuperación del horizonte $\mathrm{A}$ (materia orgánica) y 


\section{Bioetnia Volumen 7 № 1 (enero-junio), 2010}

la implementación de medidas de protección que disminuyan la radiación directa de los rayos del sol al suelo y con esto el aumento de la temperatura del suelo, para lo cual es necesaria una recuperación rápida de la estructura vegetal..

Componente vegetación: caracterización parcial de la vegetación. Las observaciones de campo en especies forestales y los muestreos al azar de la flora asociada con ecosistemas presentes en el área de estudio, permitieron visualizar los impactos a los que han sido sometidos los bosques, que reflejan la pérdida de diversidad en cuanto al número de especies florísticas se refiere, que al compararla con áreas adyacentes resulta ser muy bajo. Un muestreo rápido de vegetación en el remanente de bosque del área degradada por minería conocida como El Colegio, arrojó un total de 24 familias, distribuidas en 40 géneros y 57 especies (Tabla 3). Las familias más representativas fueron: Arecaceae con diez especies, seguida por Araceae con ocho especies, por último se encontró a Clusiaceae y Melastomatacea con cinco especies cada una y Moracea con tres especies. A lo largo del recorrido se evidenciaron procesos de sucesión natural con estadíos medios de las mismas y remanentes de rodales de especies forestales como carrá, algarrobo y cedro entre otras. Lo anterior revela que con la extracción minera se causó un grave daño al bosque, porque éste sufrió una fuerte fragmentación, así como la pérdida de especies que en la actualidad se encuentran listadas en los libros rojos colombianos en alguna categoría.

En los sitios directos donde se realizó la explotación minera se observaron diferentes grupos de plantas que por su naturaleza pueden dominar estos espacios; éstas se distribuyen de la siguiente manera 18 familias, 30 géneros y 37 especies (Tabla 4).

Las familias más representativas fueron: Cyperaceae con nueve especies, seguida de Melastomataceae con ocho especies y Poaceae con tres especies. Las especies que se observaron dentro del área degradada, aunque se conocen como especies pioneras por los altos requerimientos de luz y pocos requerimientos de nutrientes, se encontraron cerca a la quebrada Doña Josefa que bordea gran parte del área estudiada, lo que podría indicar que la influencia de la gran cantidad de materia orgánica que arrastra la quebrada favorece el rápido establecimiento de las especies listadas en la Tabla 4.

Los resultados anteriores muestran el efecto que causan este tipo de actividades; una vez que el bosque se utiliza en prácticas mineras, cambia significativamente su estructura y composición florística, así como el uso del mismo; lo anterior no sólo deteriora la vegetación existente sino que al mismo tiempo afecta de manera significativa la fauna presente en determinadas áreas, que tiene que migrar en busca de nuevos hábitats. Sin embargo, aunque el bosque nunca vuelve a tener las mismas funciones (durante un tiempo bastante considerable), se pueden establecer estrategias que permitan recupe- rar por lo menos algunas de las funciones que tenía anteriormente; por tanto, se recomienda la siembra de algunas especies tolerantes a este tipo de ambiente que permitirán el restablecimiento gradual de este ecosistema.

Aunque las especies que podrían utilizarse para la restauración ecológica del bosque como Ocroma piramydalys, Vismia macrophylla, Cespedecia espatulata, Cespedecia grandiflora y diferentes especies de la familia Fabaceae, consideradas como especies pioneras, no son especies muy utilizadas por las comunidades, poseen un rápido crecimiento que va a permitir la adecuación del suelo y a su vez se pueden alternar con especies como Vixa Orellana, Carica papaya y otras que se adaptan de forma fácil a ambientes fuertemente intervenidos y no son muy exigentes con los requerimientos de nutrientes. Es importante tener en cuenta que algunos autores señalan que la selección de especies está sujeta a los objetivos de uso del suelo en correspondencia con las características específicas del sitio y que las especies pioneras que invaden las zonas afectadas se deben ser considerar en primera instancia en los planes de revegetación.

Componente faunístico. La fauna silvestre de los bosques remanentes del área de estudio fue imposible de documentar en los recorridos de campo, porque la mayoría de los sitios se encuentran cerca de áreas donde aún se practica la actividad minera, y el uso continuo de un significativo número de maquinarias pesadas, produce ruidos a grandes escalas que afectan a los vertebrados y causan desorientación a las especies que ubican sus diferentes rutas y dimensiones de distribución en el hábitat a través del sonar; es así que grupos como los mamíferos voladores (quirópteros) y las aves fueron muy poco avistadas por el equipo técnico del proyecto; los roedores medianos y grandes y los ungulados no se avistaron; sin embargo, testimonios de un grupo de jóvenes cazadores afirman que antes se podían cazar muchos individuos de guaguas (Cuniculus paca), tatabros (Tayassu tajacu y Tayassu pecari), osos perezosos (Bradypus variegatus y Cholepus hoffmanni), michichí (Saquinus geofry), osos hormigueros (Tamandua mexicana y Myrmecophaga tridactyla), venados (Mazama americana y Odocoilues virginatus), pero que la desforestación, la cantidad de ruido y el olor a azogue han ahuyentado las poblaciones de estas especies. La situación es preocupante si se resalta que las especies mencionadas se encuentran en su mayoría dentro de los libros rojos de especies amenazadas de Colombia en categorías que van desde preocupación menor (LC) hasta peligro crítico (CR); estas especies por su presión de caza en el territorio nacional se encuentran en los apéndices I y II de CITES, esto muestra lo vulnerable que es la fauna silvestre ante la actividad minera en el municipio del Atrato; otras observaciones particulares en las áreas intervenidas, es la abundancia de especies generalistas en dietas y en hábitats como algunos herpetos (anfibios y reptiles), que se observaron directamen- 
Áreas degradadas por la minería en el Atrato. L Vargas et al.

Tabla 4

Especies observadas dentro del área degradada por minería "El Colegio"

\begin{tabular}{|c|c|c|c|}
\hline $\mathbf{N}^{\circ}$ & Familia & Especie & Nombre vulgar \\
\hline 1 & Apocinaceae & Mandevilla hirsuta & \\
\hline 2 & Araceae & Colocasia sp & \\
\hline 3 & & Philodendrum sp & \\
\hline 4 & Asteraceae & & \\
\hline 5 & Cecropiaceae & Cecropia peltata & Yarumo \\
\hline 6 & Cyperaceae & Cyperus of luzulae & \\
\hline 7 & & Cyperus ferax & \\
\hline 8 & & Cyperus haspan & \\
\hline 9 & & dyplacrum capitatum & \\
\hline 10 & & rhynchospora of polysianchys & \\
\hline 11 & & rhynchospora of pudera & \\
\hline 12 & & scleria mitis & \\
\hline 13 & & Davilla Kunthii & \\
\hline 14 & & Doliocarpus sp & \\
\hline 15 & Escrofulariaceae & & Hierba de sapo \\
\hline 16 & Eriocaulaceae & Tonina fluviatilis & Destrancadora \\
\hline 17 & Euphorbiaceae & Croton chocoensis & \\
\hline 18 & Fabaceae & Desmodium adscendens & Osodo \\
\hline 19 & Lamiaceae & Icthys & \\
\hline 20 & & Hyptis verticillata & Mastranco \\
\hline 21 & Malvaceae & Sida rhombifolia & Escobabosa \\
\hline 22 & Melastomataceae & Acisanthera quebrarata & \\
\hline 23 & & Aciotis & \\
\hline 24 & & Aciotis & \\
\hline 25 & & Clidemia rubra & \\
\hline 26 & & Clidemia crunelata & \\
\hline 27 & & Leandra sp & \\
\hline 28 & & Tococa guianensis & \\
\hline 29 & Mimosaceae & Mimosa púdica & Dormilona \\
\hline 30 & Oenoteraceae & Ludwigia sp & \\
\hline 31 & Poacea & Homolepis aturensis & \\
\hline 32 & & Ischaemum latifolium & \\
\hline 33 & & Litachne $s p$ & \\
\hline 34 & Piperaceae & Piper & \\
\hline 35 & & Piper aduncum & Cordoncillo \\
\hline 36 & Rubiaceae & Psychotria poeppigiana & \\
\hline 37 & Verbenaceae & Stachytarpheta cayenensis & Verbena morada \\
\hline
\end{tabular}




\section{Bioetnia Volumen 7 № 1 (enero-junio), 2010}

te en campo durante los trabajos diurnos. Estos herpetos Gekos y Anollis para reptiles y bufos, salamandras y cecilias para anfibios fueron frecuentes y abundantes por la exposición al sol y la formación de diferentes cuerpos de aguas propios para su reproducción .

Componente ecosistémico. Los análisis de los componentes biofísicos (agua, suelo, flora y fauna) que en conjunto forman el ecosistema, muestran la gran alteración de las dinámicas sistémicas del área; se observa claramente a nivel macro un rompimiento de la matriz original del bosque que se encuentra fragmentado en gran proporción, lo que incide directamente en el mantenimiento y viabilidad de las poblaciones de fauna silvestre. También los procesos naturales de los ecosistemas como el mantenimiento de redes tróficas han sido alterados a tal punto que es casi imposible conseguir una secuencia trófica natural en el lugar; los microambientes también se alteraron como es el caso de la hojarasca descompuesta, hábitat predilecto de la artropofauna, organismos encargados de los procesos de descomposición y ciclaje de nutrientes que permiten el mantenimiento del bosque desde los eslabones más bajos de la cadena trófica; este elemento importante de nuestro ecosistema ha desaparecido con la pérdida de gran parte del suelo del lugar.

Los ecosistemas acuáticos (ríos, riachuelos y quebradas) han sufrido los daños más severos que en algunos casos pueden ser irreparables; el solo hecho de haber desarticulado la red de drenajes naturales, cambia en términos generales la estructura y composición de la ictiofauna del lugar, llegando a casos extremos como los de la quebrada Santa Bárbara en la mina Mandinga que no contiene peces debido a la alta sedimentación que impide su desarrollo.

El microclima, la variable dependiente de todos los organismos presentes en el área de estudio y que a su vez regula la permanencia y abundancia de las comunidades biológicas de este lugar, se ha modificado también, y elementos importantes de éste como la temperatura interna del bosque y el suelo, la humedad relativa y la exposición directa de los rayos del sol, que se autorregulan en condiciones normales, han cambiado totalmente con la deforestación de los bosques para la realización de la actividad minera. Vale la pena resaltar que los efectos no son sólo a nivel de composición y estructura de los ecosistemas del área, la función también se afectó y procesos como la captación de $\mathrm{CO}_{2}$ y el secuestro de carbono por la retención de biomasa, que en conjunto contribuyen con la mitigación del cambio climático se redujeron con la modificación de los ecosistemas.

Es importante mencionar que no sólo se está poniendo en peligro la diversidad y dinámica ecosistémica del lugar, sino que se está rompiendo el linaje coevolutivo que ha permitido que durante millones de años estos dos elementos compartan una dimensión de su nicho para asegurar su permanencia en un ecosistema como éste.

\section{CONCLUSIONES}

En el municipio del Atrato existen más de 30 áreas degradadas por la minería en estado de abandono, lo que exige que las autoridades ambientales y los entes territoriales establezcan alternativas efectivas para el control y seguimiento de esta actividad para frenar el impacto desmedido en los ecosistemas de la zonas.

El recurso hídrico presenta el mayor impacto negativo de la actividad minera, pues se lograron evidenciar fuertes desviaciones de cauces, gran cantidad de represamientos de cuerpos de aguas lóticas, cambios en los procesos naturales de escorrentías a causa de la modificación del relieve natural del suelo y una alta sedimentación por el aporte de sólidos procedentes del lavado de los sustratos del suelo, lo que genera además una disminución acelerada de la oferta hídrica de la zona y afectación en todos los elementos que se encuentran adjuntos a la red.

Para el logro del mejoramiento de los suelos, se hacen necesarios procesos de adecuacion de terrenos porque por la práctica de la actividad minera se encuentran distribuidos de forma irregular, construcción de redes de drenaje para la evacuación de grandes cantidades de agua retenidas en los mismos y recuperación de la capa orgánica mediante implementación de cultivos con especies de la zona que ayuden además a disminuir la radiacion directa de los rayos del sol.

La actividad minera cambia significativamente la estructura del bosque y su composición florística, así como el uso del mismo, lo que impacta no sólo la vegetación existente sino que también afecta significativamente la fauna presente, que migra en busca de nuevos hábitats.

Con la actividad minera sin control ambiental no sólo se pone en peligro la diversidad y dinámica ecosistémica del lugar, sino que se rompe el linaje coevolutivo de muchas especies.

\section{LITERATURA CITADA}

Alcaldía Municipal del Atrato. 2001. Plan de Desarrollo Municipal del Atrato "Al Servicio de la Gente». Yuto, Chocó. Alcaldía Municipal de Atrato, 2001-2003.

Alcaldía Municipal del Atrato. 2001. Programa Agropecuario Municipal «Al Servicio de la Gente». Yuto, Chocó. Alcaldía Municipal del Atrato, 20012003.

Arango, M. S. D., M. T. Saldarriaga. 1997. Monografia del Chocó. Bogotá, DC: Editorial Autores Chocoanos; 220 p.

CORPOICA-DNP-FONADE. 1998. Caracterización de los sistemas de producción agropecuarios de la región pacífica colombiana. Bogotá, DC: CORPOICA-DNP-FONADE; 156 p.

Departamento Administrativo Nacional de Estadística (DANE). 2003. Censo. Estadísticas vitales. [Acceso 6 de octubre de 2009]. URL disponible en: http://www.dane.gov.co/files/investigaciones/fichas/poblacion/ ficha_vitales.pdf

Hanna Instruments. Manganeso, nitrato, nitrito, oxígeno disuelto. Hanna Instruments Argentina S.A. En línea. URL disponible en: http:// 


\section{Áreas degradadas por la minería en el Atrato. L Vargas et al.}

www.hannaarg.com/productos/Catalogo/Test-kits/Otros/c_3810.pdf Ministerio del Medio Ambiente, Instituto Geográfico Agustín Codazzi. 2000. Zonificación ecológica de la región pacífica colombiana. Bogotá, DC: Ministerio del Medio Ambiente, Instituto Geográfico Agustín Codazzi (IGAC); $286 \mathrm{p}$.

Ramírez, F. 2007. Tierra y minería, el conflicto en Colombia. Revista Semillas. 2007; 32/33. Bogotá DC. 10 p. En línea. URL disponible en: http:// Www.semillas.org.co/sitio.shtml?apc=e1b-20155114-20155 114 HYPERLINK «http://www.semillas.org.co/sitio.shtml?apc=e1b20155114-20155114\&x=20155119»\&HYPERLINK«http://www . semillas.org.co/sitio.shtml?apc $=$ e $1 \mathrm{~b}-20155114-20155114 \& \mathrm{x}=20$ $155119 » \mathrm{x}=20155119)$

Rivas, J. 2005. Historiografía del departamento del Chocó. $2^{\mathrm{a}}$ ed. Quibdó: Promotora Editorial de Autores Chocoanos; p. 178.

Stumm W., Morgan J. 1988. Aquatic chemistiy: an introduction emphasizing chemical equilibria in natural waters. New York: Wiley-Interscience. Current Contents N 41. 583 p

Unidad Regional de Planificación Agropecuaria, Ministerio de Agricultura, Secretaría de Agricultura. 1989. Diagnóstico sector agropecuario-recursos naturales departamento del Chocó. Quibdó: Gobernación del Chocó. $258 \mathrm{p}$. 